\title{
Preoperative Conformal Radiotherapy Concurrently with Paclitaxel/Carboplatin in Gastric Cancer
}

\author{
Shimaa Ahmed1, Mayada Fawzy ${ }^{2}$, Khalid Rezk ${ }^{3 *}$, Wessam Elshrif ${ }^{4}$, Mohamed Alaa5, \\ Mohamed M. H. Abd Ellah ${ }^{6}$
}

${ }^{1}$ Radiation Oncology, South Egypt Cancer Institute, Assuit University, Assiut, Egypt

${ }^{2}$ Medical Oncology, South Egypt Cancer Institute, Assuit University, Assiut, Egypt

${ }^{3}$ Surgical Oncology, South Egypt Cancer Institute, Assuit University, Assiut, Egypt

${ }^{4}$ Clinical Oncology, Cairo University, Cairo, Egypt

${ }^{5}$ Clinical Oncology, Assuit University, Assiut, Egypt

${ }^{6}$ Diagnostic Radiology, South Egypt Cancer Institute, Assuit University, Assiut, Egypt

Email: shimaayoussif04@gmail.com, mayadaelzohri@gmail.com, ^rezk.khalid@gmail.com,wessam_elsherief@yahoo.com,

Malaaassiut@gmail.com,dr_m_hamdy2006@hotmail.com

How to cite this paper: Ahmed, S., Fawzy, M., Rezk, K., Elshrif, W., Alaa, M. and Abd Ellah, M.M.H. (2018) Preoperative Conformal Radiotherapy Concurrently with Paclitaxel/Carboplatin in Gastric Cancer. Journal of Cancer Therapy, 9, 503-515. https://doi.org/10.4236/jct.2018.96041

Received: May 11, 2018

Accepted: June 22, 2018

Published: June 25, 2018

Copyright (c) 2018 by authors and Scientific Research Publishing Inc. This work is licensed under the Creative Commons Attribution International License (CC BY 4.0).

http://creativecommons.org/licenses/by/4.0/

\section{(c) (i) Open Access}

\begin{abstract}
Background and objectives: Surgery is the primary therapy for localized gastric cancer, but even with the best results only 40\% 5-year survival can be achieved with the use of postoperative adjuvant chemoradiotherapy. Preoperative therapy might help increase the R0 resection rate, which is an independent predictor of 5-year OS. Our study hypothesized that the concurrent combination of carboplatin-paclitaxel with radiation therapy would result in a pathological CR rate, which will be in turn associated with OAS and DFS benefits. Patients and methods: prospective phase II study included 32 patients with locally advanced gastric adenocarcinoma including gastroesophageal junction who received a combination of neoadjuvant conformal radiotherapy concurrently with carboplatin-paclitaxel followed by surgery. Results: Pathological CR and R0 resection rates were $18.8 \%$ and $75 \%$ respectively. With a median follow up of 24 months, 2 years disease-free survival was $28.1 \%$ and overall survival was $51.3 \%$. The regimen was tolerated with neither grade 4 toxicities nor deaths. Conclusion: Neoadjuvant radiotherapy concomitant with carboplatin-paclitaxel chemotherapy is a well-tolerated approach for patients with locally advanced gastric adenocarcinoma resulting in significant pathological CR and R0 resection margins as reflected by the good DFS and OS.
\end{abstract}




\section{Keywords}

Gastric Adenocarcinoma, Neoadjuvant, Paclitaxel-Carboplatin, Chemoradiotherapy, Pathologicalcr-R0

\section{Introduction}

Postoperative concurrent chemoradiation (CCRT) or preoperative chemotherapy is usually the standard treatment for resectable gastric cancer, multiple randomized trials showed survival benefit as in INT-0116 and MAGIC trials [1] [2]. The perioperative chemoradiotherapy (CRT) has a well-established role in esophageal and rectal cancer [3] [4]. In gastroesophageal junction (GEJ) tumours, CROSS trial [3] in which preoperative CRT used it showed survival benefit more than perioperative chemotherapy [5]. The rationale for using perioperative CRT that the radiation field is small and accurate [6] better chemotherapy deliver with intact tumour vasculature also sterilize surgical field and eradicate subclinical metastasis [7]. Two trials have studied the role of the perioperative CRT in potentially resectable gastric tumours; one was done by Eastern cooperative oncology group (E7296), the other was done by RTOG (9904). Both used two cycles of induction chemotherapy followed by the perioperative CRT, RTOG trial achieved pathological CR more than 20\% [8]. Valenti et al. studied 72 randomized patients with advanced gastric cancer divided into two groups; the first treated with perioperative chemotherapy and the second treated with perioperative CRT. They found a more major pathological response in perioperative CRT ( $47.6 \%$ vs $13.3 \%, p=0.0024)$ with no difference regarding complication [9].

Paclitaxel is a well know radiosensitizer [10] that has proven effective in gastric cancer treatment [11], [12] as well as carboplatin combination [13].

This prospective study aimed to evaluate the clinical and pathological responses as well as the safety of preoperative conformal radiotherapy concurrently with paclitaxel and carboplatin in locally advanced gastric and gastroesophageal adenocarcinoma taking into consideration the overall survival and disease-free survival.

\section{Patients and Methods}

This current prospective phase II study was approved by our institutional review board and conducted at the radiation oncology and clinical oncology departments at our university hospital in the period from January 2014 to December 2017. For this study 32 patients with gastric and GEJ adenocarcinoma, all patients gave informed consent upon participating in the study.

Inclusion criteria included histologically proven non-metastatic adenocarcinoma of the stomach or gastroesophageal junction, Patients with T3, T4A, N2 or N3 according to AJCC staging system 2010 (version 7.0) [14], Patients age $\geq 18$ and $\leq 70$ years; ECOG performance status $\leq 2$ in addition to normal laboratory 
functions.

Exclusion criteria included distant metastasis, patient stage T1 and N0 or N1; T2 or N0 or N1, Patients with a history of other previous malignancies, patients with peripheral neuropathy, surgically unfit patients as well as pregnant women.

\subsection{Pretreatment Evaluation}

The pretreatment evaluation included careful medical history taking, physical examination, and laboratory investigations (complete blood picture, liver and kidney functions and electrolytes), CT chest and abdomen as well as upper endoscopy. Clinical staging could be further determined [15].

\subsubsection{Preoperative Chemoradiotherapy}

Chemotherapy was given weekly during radiotherapy on Saturday as follow: preparation including granisetron $1 \mathrm{mg}$, dexamethasone $8 \mathrm{mg}$ and pheniramine maleate was administered intravenously 15 minutes infusion for each one, then paclitaxel $50 \mathrm{mg} / \mathrm{m}^{2}$ one-hour infusion and carboplatin AUC2 over 30 minutes infusion.

\subsubsection{Radiotherapy Technique}

Patients should fast for $2-3 \mathrm{~h}$ before CT simulation and before treatments. CT scans with the intravenous contrast of $3 \mathrm{~mm}$ thickness obtained in the supine position using wing fixation. Radiotherapy delivered $4500 \mathrm{cGy}$ in 25 fractions within 5 weeks using 6 and $15 \mathrm{MV}$ photons. The gross tumour volume (GTV) for primary and lymph nodes as seen on CT, and endoscopic evaluation. The clinical target volume (CTV) was defined as the gross tumour volume plus a $5-\mathrm{cm}$ margin superior and inferior and $2-\mathrm{cm}$ margin radial to the tumour include entire stomach, all tumour extensions and draining lymph nodes (perigastric, celiac, porta hepatis, gastroduodenal, splenic, hilar, suprapancreatic, pancreatoduodenal, and paraaortic paraoesophageal, for tumour in GEJ at least 3 - 5 $\mathrm{cm}$ of distal oesophagus for tumour in GEJ and tumour in upper part of stomach. Dose limitation for organ at risk: kidney (70\% of one kidney volume $<20$ Gy and $30 \%$ of other kidney volume $<30 \mathrm{~Gy}$; liver (30\% of liver volume $<30 \mathrm{~Gy}$ mean dose $<30 \mathrm{~Gy})$, spinal cord $\left(\mathrm{D}_{\max }<45 \mathrm{~Gy}\right)$.

\subsubsection{Evaluation of Toxicities and Response to Chemoradiation}

An attending physician evaluated each patient on a weekly basis during concurrent chemoradiation radiation for the toxicity assessment according to CTCAEv4.0.

Assessment of response and re-staging were done three weeks after the chemoradiation by clinical evaluation and CT chest and abdomen.

\subsubsection{Surgery}

All non-metastatic patients were referred to the surgical department, where laparoscopy was done followed by radical surgery according to the tumour site. 


\subsubsection{Pathological Assessment}

Postoperative pathological assessment of the surgical margin was defined as R0 (microscopically negative), R1 (microscopically positive) or R2 (gross residual).

The estimated percentage of vital residual tumour cells (VRTCs) was evaluated. The degree of histopathological regression was classified into 4 categories [16]: grade I, $>50 \%$ VRTCs stable disease (no response); grade II, $10 \%$ to $50 \%$ VRTCs partial response (PR); grade III, $<10 \%$ VRTCs nearly complete response (NCR); and grade IV, complete response (pCR). Grades III and IV were considered as major histomorphologic response compared with grades I and II constituting a minor histopathologic response.

\subsection{Statistical Methods}

Survival time calculation from diagnosis to last follow up for disease-free (DFS) and overall survivals (OAS) according to Kaplan-Meier [17].

\section{Results}

Patients' demographics and tumour characteristics are summarized in Table 1. All patients received their preoperative chemoradiotherapy courses completely without interruption with good tolerance.

After chemoradiation patients underwent re-staging by CT chest, abdomen and pelvis. Table 2 represents tumour and nodal downstaging staging before and after chemoradiation where the number of patients who had tumour size $\leq$ T2 increased from 6 (18.8\%) patients before treatment to 20 patients (62.5\%) after treatment, however, patients with T3 andT4 tumours decreased from 26 $(81.1 \%)$ to $12(37.5 \%)$ patients. Nodal involvement $\leq \mathrm{N} 1$ showed an increase from $16(50 \%)$ patients before treatment to $27(84.4 \%)$ after treatment, on the contrary extensive nodal involvement ( $\mathrm{N} 2$ or N3) decreased from $16(50 \%)$ patients pre-treatment to $5(12.5 \%)$ patients after treatment.

All patients were referred to surgery where laparoscopy and exploration were performed. Laparoscopy revealed peritoneal metastasis in six patients, to whom surgery was cancelled. The remaining 26 patients underwent radical surgery (Table 3) with 24 (75\%) patients achieved negative microscopic margin (R0) and only 2 patients had microscopically positive margin (Table 4 ).

After chemoradiation 6 patients showed pCR, 5 patients NCR, 9 patients PR and 6 patients showed no response (Table 5), association between both pCR and NCR revealed high incidence of complete response in early tumour and nodal stage (Table 6).

All patients tolerated preoperative chemoradiation without developing grade 4 toxicities and no deaths related to chemoradiation or surgery were reported. Grade 3 haematological toxicities requiring intervention were found in 2 patients with anaemia treated by transfusion of packed RBCs, 2 patients with neutropenia less than 500 treated by hospitalization and antibiotics, and one patient with thrombocytopenia. 
Table 1. Patients' and tumour characteristics.

\begin{tabular}{|c|c|c|}
\hline Character & \multicolumn{2}{|c|}{ NO } \\
\hline \multicolumn{3}{|l|}{ Age } \\
\hline Media & \multicolumn{2}{|c|}{51} \\
\hline Range & \multicolumn{2}{|c|}{$38-64$} \\
\hline \multicolumn{3}{|l|}{ Sex } \\
\hline Male & 17 & 53.1 \\
\hline Female & 15 & 46.9 \\
\hline \multicolumn{3}{|l|}{ PS } \\
\hline 0 & 9 & 28.1 \\
\hline 1 & 20 & 62.5 \\
\hline 2 & 3 & 9.4 \\
\hline \multicolumn{3}{|l|}{ tumour } \\
\hline $\mathrm{T} 2$ & 6 & 15.6 \\
\hline T3 & 23 & 66.7 \\
\hline $\mathrm{T} 4 \mathrm{~A}$ & 3 & 9.4 \\
\hline \multicolumn{3}{|l|}{ Node } \\
\hline N1 & 16 & 50 \\
\hline $\mathrm{N} 2$ & 12 & 37.5 \\
\hline N3 & 4 & 12.5 \\
\hline \multicolumn{3}{|c|}{ Primary tumour Site } \\
\hline GEJ & 6 & 18.7 \\
\hline Proximal & 10 & 31.3 \\
\hline Distal & 12 & 37.5 \\
\hline Lenitis & 4 & 12.5 \\
\hline \multicolumn{3}{|c|}{ Histology grade } \\
\hline 1 & 3 & 9.4 \\
\hline 2 & 20 & 62.5 \\
\hline 3 & 9 & 28.1 \\
\hline
\end{tabular}

Table 2. Tumour and nodal stage before and after chemoradiation.

\begin{tabular}{ccc}
\hline stage & before & after \\
\hline tumour stage & 0 & 6 \\
T0 & 0 & 8 \\
T1 & 6 & 6 \\
T2 & 23 & 11 \\
T3 & 3 & 1 \\
T4A & & \\
Node stage & 0 & 15 \\
N0 & 16 & 12 \\
N1 & 12 & 5 \\
N2 & 4 & 0 \\
N3 & & \\
\hline
\end{tabular}


Table 3. Type of surgical procedure among all 32 patients received preoperative chemoradiation.

\begin{tabular}{ccc}
\hline Types & No & $\%$ \\
\hline Total Gastrectomy & 13 & 40.6 \\
Partial Gastrectomy & 8 & 25 \\
Esphagogastrectomy & 5 & 15.6 \\
Laparoscopy Only & 6 & 18.8 \\
Total & 32 & 100 \\
\hline
\end{tabular}

Table 4. Pathological margin status among 32 patients after preoperative chemoradiation.

\begin{tabular}{ccc}
\hline Margin status & No & $\%$ \\
\hline R0 & 24 & 75 \\
R1 & 2 & 6.2 \\
R2 & 6 & 18.8 \\
Total & 32 & 100
\end{tabular}

Table 5. Pathologic response among 32 patients after preoperative chemoradiation.

\begin{tabular}{ccc}
\hline Response & No & $\%$ \\
\hline CR & 6 & 18.8 \\
NCR & 5 & 15.6 \\
PR & 9 & 28.1 \\
SD & 6 & 18.8 \\
PD & 6 & 18.8 \\
Total & 32 & 100 \\
\hline
\end{tabular}

Table 6. Association between pathological response and tumour stage among 32 patients.

\begin{tabular}{cccc}
\hline & Total patients & Complete response & $\%$ \\
\cline { 2 - 3 } & No & No & 18.8 \\
All & 32 & 6 & \\
tumour stage & & 3 & 59 \\
T2 & 6 & 3 & 13 \\
T3 & 23 & 0 & 0 \\
T4a & 3 & & \\
Nodal stage & & 4 & 25 \\
N1 & 16 & 2 & 16.7 \\
N2 & 12 & 0 & 0 \\
N3 & 4 & & \\
\hline
\end{tabular}


Gastrointestinal toxicities mainly grade 1 and 2 were found in only 3 patients, who were admitted with intravenous fluid administration because of severe vomiting (Table 7).

\section{Survival}

With a median follow up 24 months (range 7 - 41 months), disease-free survival (DFS) was $28.1 \%$ (Figure 1), and only CR and PR are significant factors.

Two years overall survival (OAS) for all 32 patients was 51.3\% (Figure 1), none of the six patients with pCR died during the median follow up period, while 2 years OAS for patients who did without achieving CR (26 patients) was $37.9 \%$ ( $\mathrm{P}=0.0118$ ) (Figure 2 ). Microscopically negative margin ( $\mathrm{R} 0$ ) is another significant factor for survival as 2 years OAS was $65 \%$ for patient achieved negative microscopic margin while none of the remaining 8patients [6] patients had peritoneal deposits and 2 patients had positive microscopic margin], which did not achieve R0 survived for 2 years (Figure 3 ).

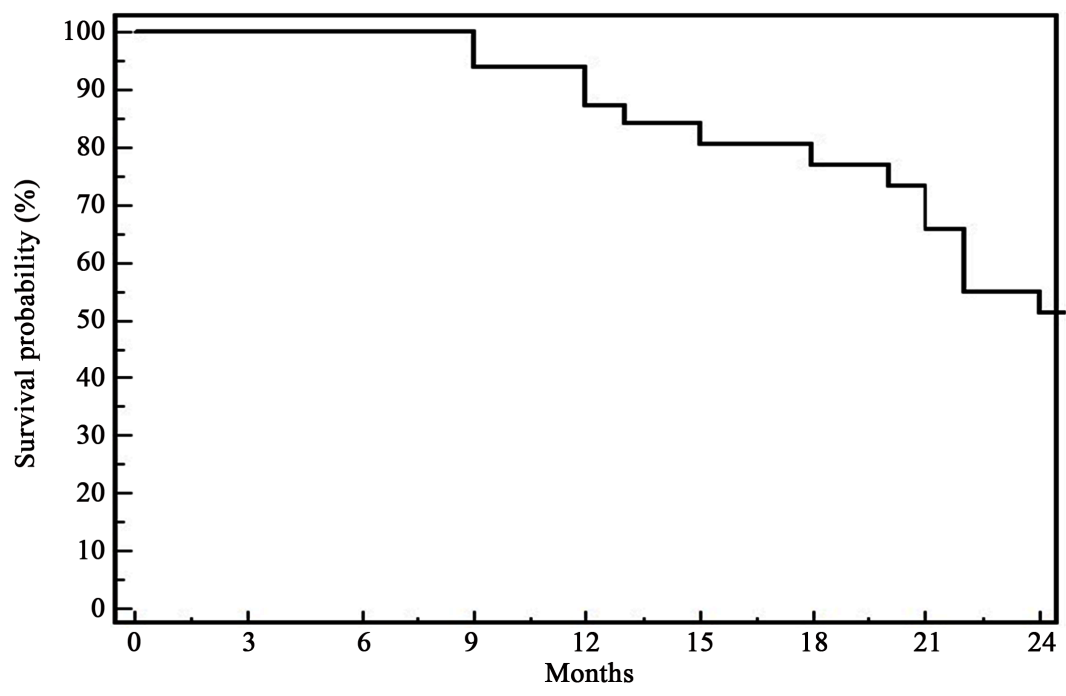

Figure 1. OAS for 32 patients received preoperative chemoradiation.

Table 7. Acute toxicity among 32 patients received preoperative chemoradiation.

\begin{tabular}{|c|c|c|c|c|c|}
\hline \multicolumn{2}{|c|}{ Toxicity } & \multirow{2}{*}{$\begin{array}{c}\mathrm{G} 1 \\
15\end{array}$} & \multirow{2}{*}{$\frac{\mathrm{G} 2}{3}$} & \multirow{2}{*}{$\begin{array}{c}\text { G3 } \\
2\end{array}$} & \multirow{2}{*}{$\begin{array}{c}\mathrm{G} 4 \\
0\end{array}$} \\
\hline & Anemia & & & & \\
\hline \multirow[t]{4}{*}{ Hematological toxicities } & Neutropenia & 18 & 5 & 2 & 0 \\
\hline & Thrombocytopenia & 16 & 4 & 1 & 0 \\
\hline & Diarrhea & 6 & 1 & 0 & 0 \\
\hline & Anorexia & 3 & 10 & 1 & 0 \\
\hline \multirow[t]{3}{*}{ GIT toxicities } & Nausea \& vomiting & 16 & 8 & 3 & 0 \\
\hline & Dysphagia & 10 & 2 & 1 & 0 \\
\hline & Mucositis & 1 & 0 & 0 & 0 \\
\hline \multicolumn{2}{|c|}{ Neurotoxicity } & 15 & 2 & 0 & 0 \\
\hline \multicolumn{2}{|c|}{ Weight loss } & 18 & 3 & 0 & 0 \\
\hline \multicolumn{2}{|c|}{ Fatigue } & 26 & 3 & 1 & 0 \\
\hline
\end{tabular}




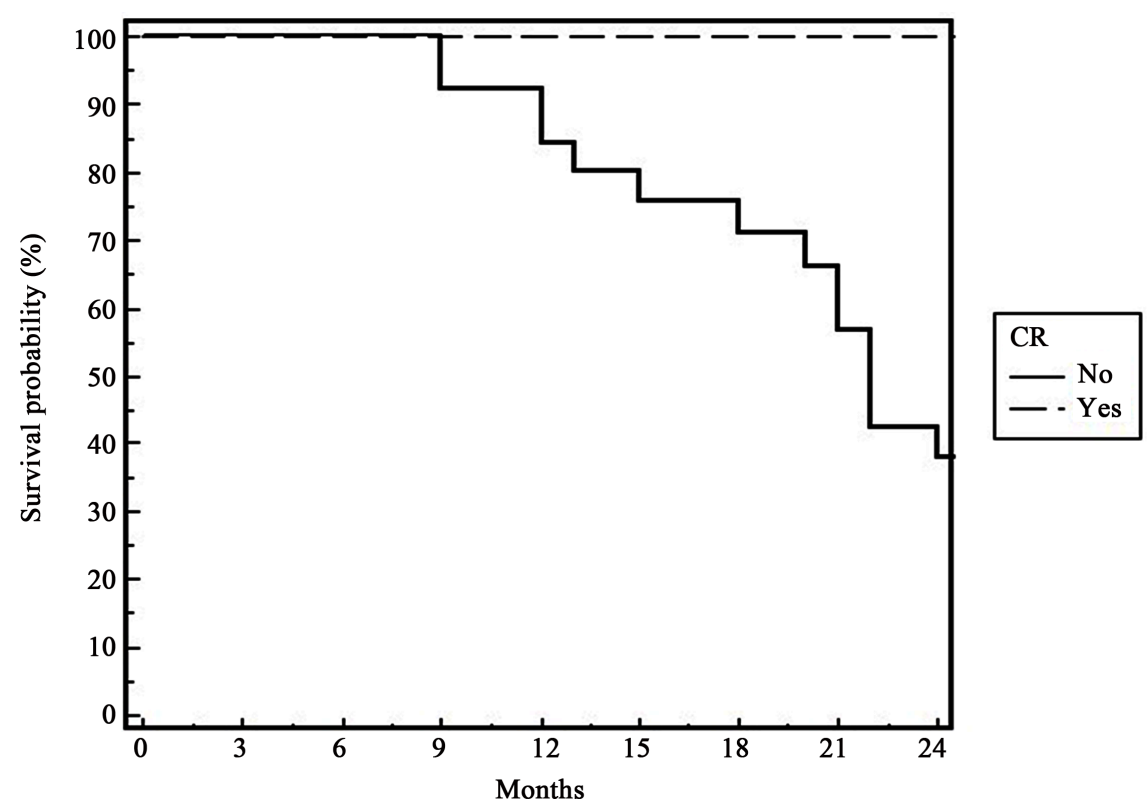

Figure 2. OAS according to CR achievements for 32 patients received preoperative chemoradiation.

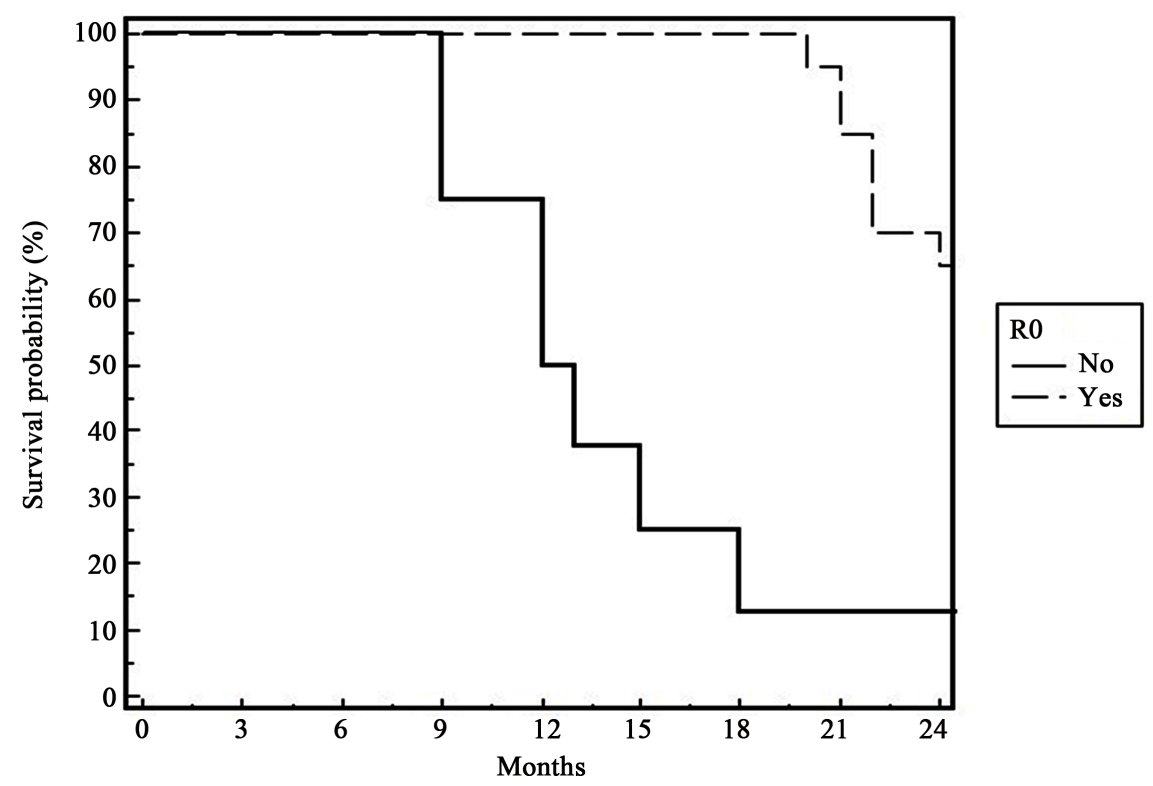

Figure 3. OAS according to negative margin achievements for 32 patients received preoperative chemoradiation.

\section{Discussion}

Surgery is the primary therapy for localized gastric cancer, but even with the best results, only $40 \% 5$-year survival could be achieved with the use of postoperative adjuvant chemoradiotherapy. Preoperative therapy might help increasing the R0 resection rate, which is an independent predictor of the 5-years OAS [18]. A randomized study in which an increase in the $\mathrm{R} 0$ resection rate was reported with the use of preoperative chemotherapy, furthermore suggesting that this 
strategy might be valuable in improving important end points [2].

The advantages of neoadjuvant approach; particularly chemotherapy in the treatment of aggressive solid tumour malignancies have been reported and some were proven. Early treatment of distant microscopic disease, the ability to evaluate in vivo response to therapy, and the potential for tumour down staging to enhance respectability are translated to better outcomes [19].

Neoadjuvant strategy has already increased the likelihood of completing multimodality therapy, particularly when surgical management is associated with significant morbidity and complications which may delay or prevent adjuvant therapy [17].

Application of radiotherapy in the neoadjuvant setting has several advantages. The presence of an intact tumour and preserved normal anatomy facilitates treatment planning and may limit toxicity to adjacent organs. On the other hand, adjuvant radiotherapy mandates larger treatment fields with the risk of increased toxicity. Such advantages paved the way to better outcomes in rectal and oesophagal cancer. In rectal cancer, preoperative CRT decreases locoregional recurrence compared to postoperative CRT. Preoperative, compared with postoperative, CRT also decreases the incidence of grades 3 and 4 adverse events and long-term toxic effects, and improves sphincter preservation [4].

In oesophagal cancer, neoadjuvant CRT showed better outcome in terms of DFS and OAS compared with surgery alone. The success of preoperative CRT in oesophagal and rectal cancer may be attributed to the anatomic location of the esophagus and rectum in enclosed spaces where the ability to achieve a negative radial margin may be challenging and treatment can be administered with less risk of toxicity to adjacent organs [3].

The concept that a neoadjuvant approach may deprive a subset of patients of curative therapy ending in progression prior to surgery is totally incorrect. However, identification of patients who could be spared from a potentially morbid non-curative resection (i.e., those that would recur distantly early point) is an additional advantage of a neoadjuvant approach. There is a potential for treatment-related toxicity that may delay or prevent surgical therapy in patients with the curable disease. Hopefully, the poor outcomes associated with surgery alone for all but early-stage gastrointestinal malignancies alleviate such concerns.

Generally, the approach where patients with the clinical early-stage disease are treated with surgery first and those with locally advanced disease receive neoadjuvant therapy is nearly applied in most centres worldwide.

The advantages of neoadjuvant therapy are generally applicable to the treatment of gastric cancer (GC). First, even with an R0 resection, local and systemic recurrence is common; predicting that early treatment of occult microscopic disease could decrease recurrence possibility.

Second, gastrectomy for GC is associated with substantial morbidity. Neoadjuvant therapy gives the chance for multimodality therapy completion. 
Third, in vivo testing of neoadjuvant treatment response, which is directly related to long-term outcome; showed that a favourable response may, therefore, justify an aggressive surgical approach [20].

Still, the value of preoperative CRT therapy for patients with resectable gastric cancer is uncertain and is the subject of an ongoing international prospective phase III randomized trial [21].

In the current stud, our goal was to evaluate clinical and pathological response, and safety of preoperative conformal radiotherapy concurrently with paclitaxel and carboplatin in locally advanced gastric and gastroesophageal adenocarcinoma.

In our study, Pathological CR and R0 resection rates were $18.8 \%$ and $75 \%$ respectively. In our study with a median follow up 24 months 2 years disease-free survival was $28.1 \%$. Although CR and PR were the only significant factors, they were not highly significant compared to the other factors because of the low number of patients.

With a median follow up of 24 months, (range $7-41$ months), 2 years OAS was $51.3 \%$ (Figure 4). None of six patients with pCR died during the median follow-up period of 2 years OAS for patients, who did not achieve CR was 37.9\% (Figure 1). R0 is another significant factor for survival as 2 years OAS was $65 \%$ (Figure 2). Four patients achieved negative microscopic margin while none of the remaining eight patients [six patients had peritoneal deposits and 2 patients had positive microscopic margin], who did not achieve R0 survive for 2 years.

The regimens listed in our protocol are derived from the phase III trial, cross trial, that has included patients with adenocarcinoma of the esophagus and/or esophago-gastric junction (EGJ), in this trial OAS and DFS were significantly better in the neoadjuvant setting thus making this regimen a new standard of care in distal oesophagal and EGJ tumours (7).

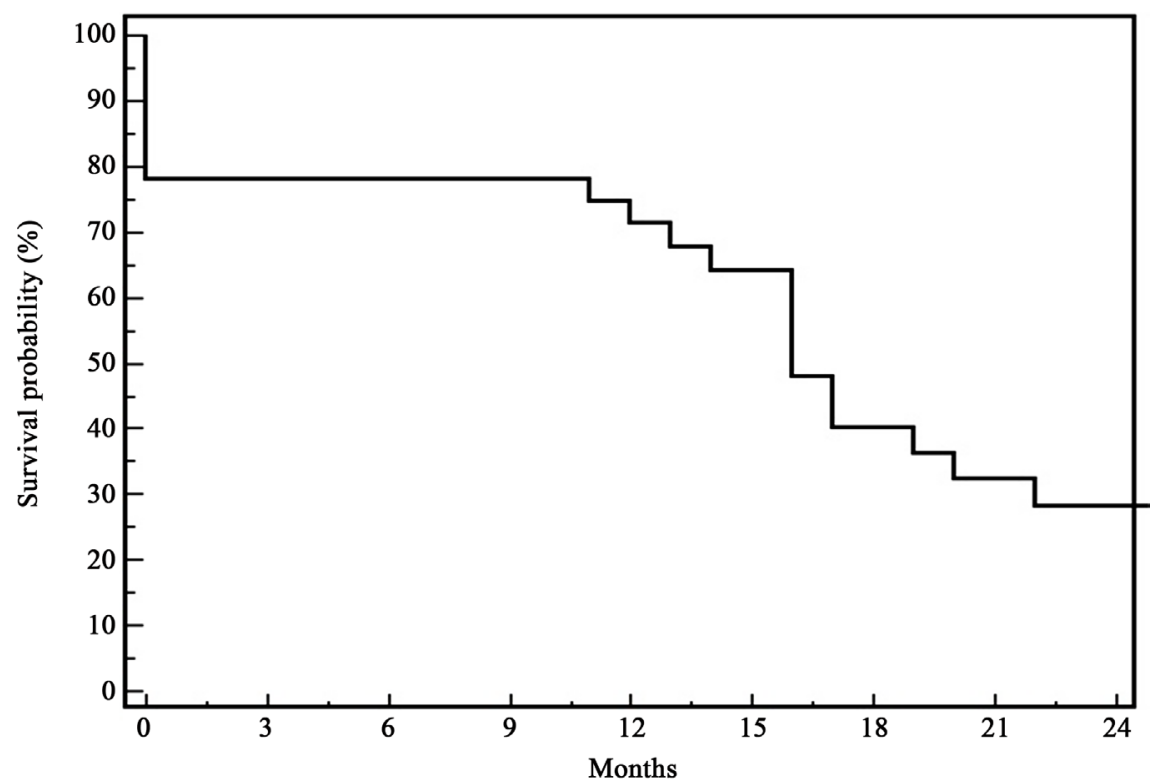

Figure 4. DFS among 32 cases underwent preoperative chemoradiation. 
In a pilot study, Lowy and colleagues assessed the feasibility of preoperative chemoradiation (45 Gy of external beam RT with concurrent continuous infusion of fluorouracil) followed by surgery and intraoperative RT (IORT; 10 Gy) in the treatment of patients with potentially respectable gastric cancer. In this study 93\% completed protocol where in our study all the patients completed preoperative treatment, in his study four patients $17 \%$ progressed and surgery was omitted and that was concordant with our study, where 6 patients $18.8 \%$ didn't do radical surgery, where the rate of pathological CR was doubled in our study, where 6 patients $18.8 \%$ in comparison to 2 patients $11 \%$, this might be explained by different chemotherapy protocols [22].

Similarly, in the RTOG 9904 phase II trial of preoperative paclitaxel-based chemoradiation, the pathological CR and R0 resection rates were $26 \%$ and $77 \%$ respectively and the survival was better in patients achieved pathological CR, which was in accordance with our results [23].

Moreover, in another phase II trial conducted by Ajani et al., patients received two cycles of paclitaxel-based chemoradiation, and R0 resection was feasible in $78 \%$ of patients. A pathological CR rate of $20 \%$ and a pathological PR rate of $15 \%$ ( $<10 \%$ residual cancer cells in the resected specimen) were noted [20].

OS and DFS were significantly correlated with R0 resection, pathological response, dissected pathologically positive $\mathrm{LN}$, and post surgery $(\mathrm{T})$ stage. Interestingly, Ajani et al. reported that OAS and DFS were significantly correlated with comparable factors again these results augment our results about the importance of pathological CR as an important prognostic factor in GC [20].

In our study, all patients tolerated preoperative chemoradiation without developing grade 4 toxicities and deaths related to chemoradiation or surgery.

On the other hand, in the RTOG 9904 study, grade 4 toxicity was reported in $21 \%$ patients and there were no treatment-related deaths [23].

Additionally, Ajani et al. reported $24 \%$ grade 4 chemoradiation toxicity [20]. Again this different incidence of grade 4 toxicity with less toxicity in our study might be related to different chemotherapy regimens and better supportive care than older studies.

\section{Conclusion}

Neoadjuvant radiotherapy concomitant with chemotherapy paclitaxel-carboplatin is a well-tolerated neoadjuvant therapy for patients with locally advanced gastric adenocarcinoma resulting in significant pathological CR and R0 resection margins reflected on a better DFS and OS.

\section{References}

[1] McLoughlin, J.M. (2004) Adenocarcinoma of the Stomach: A Review. Proceedings (Baylor University. Medical Center), 17, 391-399. https://doi.org/10.1080/08998280.2004.11928001

[2] Cunningham, D., et al. (2006) Perioperative Chemotherapy versus Surgery Alone for Resectable Gastroesophageal Cancer. The New England Journal of Medicine, 355, 11-20. https://doi.org/10.1056/NEJMoa055531 
[3] van Hagen, P. et al. (2012) Preoperative Chemoradiotherapy for Esophageal or Junctional Cancer. The New England Journal of Medicine, 366, 2074-2084. https://doi.org/10.1056/NEJMoa1112088

[4] Sauer, R. et al. (2004) Preoperative versus Postoperative Chemoradiotherapy for Rectal Cancer. The New England Journal of Medicine, 351, 1731-1740.

https://doi.org/10.1056/NEJMoa040694

[5] Ychou, M., Boige, V., Pignon, J.-P., Conroy, T., Bouché, O., Lebreton, G., et al. (2011) Perioperative Chemotherapy Compared with Surgery Alone for Resectablegastroesophageal Adenocarcinoma: An FNCLCC and FFCD Multicenter phase III Trial. Journal of Clinical Oncology, 29, 1715-1721. https://doi.org/10.1200/JCO.2010.33.0597

[6] Pepek, J.M., et al. (2013) Preoperative Chemoradiotherapy for Locally Advanced Gastric Cancer. Radiation Oncology, 8, 6. https://doi.org/10.1186/1748-717X-8-6

[7] The Concurrent Chemoradiation Paradigm-General Principles. https://www.medscape.com/medline/abstract/17259930

[8] Ajani, J.A., Mansfield, P.F., Crane, C.H., et al. (2005) Paclitaxel-Based Chemoradiotherapy in Localized Gastric Carcinoma: Degree of Pathologicresponse and Not Clinical Parameters Dictated Patient Outcome. Journal of Clinical Oncology, 23, 1237-1244. https://doi.org/10.1200/JCO.2005.01.305

[9] Valenti, V., Hernandez-Lizoaín, J.L., Beorlegui, M.C., Diaz-Gozalez, J.A., Regueira, F.M., Rodriguez, J.J., et al. (2011) Morbidity, Mortality, and Pathological Response Inpatients with Gastric Cancer Preoperatively Treated with Chemotherapy Orchemoradiotherapy. Journal of Surgical Oncology, 104, 124-129.

https://doi.org/10.1002/jso.21947

[10] Safran, H., et al. (2000) Paclitaxel and Concurrent Radiation for Gastric Cancer. International Journal of Radiation Oncology, Biology, Physics, 46, 889-894. https://doi.org/10.1016/S0360-3016(99)00436-8

[11] Ajani, J.A., Ilson, D.H., Daugherty, K., Pazdur, R., Lynch, P.M. and Kelsen, D.P. (1994) Activity of Taxol in Patients with Squamous Cell Carcinoma and Adenocarcinoma of the Esophagus. Journal of the National Cancer Institute, 86, 1086-1091. https://doi.org/10.1093/jnci/86.14.1086

[12] Ajani, J.A., Fairweather, J., Dumas, P., Patt, Y.Z., Pazdur, R. and Mansfield, P.F. (1998) Phase II Study of Taxol in Patients with Advanced Gastric Carcinoma. The Cancer Journal from Scientific American, 4, 269-274.

[13] Trip, A.K., et al. (2014) Preoperative Chemoradiotherapy in Locally Advanced Gastric Cancer, a Phase I/II Feasibility and Efficacy Study. Radiotherapy \& Oncology, 112, 284-288. https://doi.org/10.1016/j.radonc.2014.05.003

[14] Washington, K. (2010) 7th Edition of the AJCC Cancer Staging Manual: Stomach. Annals of Surgical Oncology, 17, 3077-3079. https://doi.org/10.1245/s10434-010-1362-Z

[15] Halvorsen, R.A., Magruder-Habib, K., Foster, W.L., Roberts, L., Postlethwait, R.W. and Thompson, W.M. (1986) Esophageal Cancer Staging by CT: Long-Term Follow-Up Study. Radiology, 161, 147-151. https://doi.org/10.1148/radiology.161.1.3763857

[16] Schneider, P.M., et al. (2005) Histomorphologic Tumor Regression and Lymph Node Metastases Determine Prognosis Following Neoadjuvant Radiochemotherapy for Esophageal Cancer: Implications for Response Classification. Annals of Surgery, 242, 684-692. https://doi.org/10.1097/01.sla.0000186170.38348.7b

[17] Kaplan, E.L. and Meier, P. (1958) Nonparametric Estimation from Incomplete Observations Nonparametric Estimation from Incomplete Observations. Journal of the 
American Statistical Association, 53, 457-481. https://doi.org/10.1080/01621459.1958.10501452

[18] Schwartz, G.K., et al. (2009) Randomized Phase II Trial Evaluating Two Paclitaxel and Cisplatin-Containing Chemoradiation Regimens as Adjuvant Therapy in Resected Gastric Cancer (RTOG-0114). Journal of Clinical Oncology, 27, 1956-1962. https://doi.org/10.1200/JCO.2008.20.3745

[19] Ajani, J.A., et al. (1999) Enhanced Staging and All Chemotherapy Preoperatively in Patients with Potentially Resectable Gastric Carcinoma. Journal of Clinical Oncology, 17, 2403-2411. https://doi.org/10.1200/JCO.1999.17.8.2403

[20] Ajani, J.A., et al. (2005) Paclitaxel-Based Chemoradiotherapy in Localized Gastric Carcinoma: Degree of Pathologic Response and Not Clinical Parameters Dictated Patient Outcome. Journal of Clinical Oncology, 23, 1237-1244.

https://doi.org/10.1200/JCO.2005.01.305

[21] Leong, T., et al. (2015) TOPGEAR: A Randomised Phase III Trial of Perioperative ECF Chemotherapy versus Preoperative Chemoradiation plus Perioperative ECF Chemotherapy for Resectable Gastric Cancer (an International, Intergroup Trial of the AGITG/TROG/EORTC/NCIC CTG). BMC Cancer, 15, 532. https://doi.org/10.1186/s12885-015-1529-X

[22] Lowy, A.M., et al. (2001) A Pilot Study of Preoperative Chemoradiotherapy for Resectable Gastric Cancer. Annals of Surgical Oncology, 8, 519-524. https://doi.org/10.1007/s10434-001-0519-1

[23] Ajani, J.A., et al. (2006) Phase II Trial of Preoperative Chemoradiation in Patients with Localized Gastric Adenocarcinoma (RTOG 9904): Quality of Combined Modality Therapy and Pathologic Response. Journal of Clinical Oncology, 24, 3953-3958. https://doi.org/10.1200/JCO.2006.06.4840 